\title{
Pictures of complete positivity in arbitrary dimension
}

\author{
Bob Coecke and Chris Heunen
}

July 10, 2018

\begin{abstract}
Two fundamental contributions to categorical quantum mechanics are presented. First, we generalize the CPM-construction, that turns any dagger compact category into one with completely positive maps, to arbitrary dimension. Second, we axiomatize when a given category is the result of this construction.
\end{abstract}

\section{Introduction}

Since the start of categorical quantum mechanics 2], dagger compactness has played a key role in most constructions, protocol derivations and theorems. To name two:

- Selinger's CPM-construction, which associates to any dagger compact category of pure states and operations a corresponding dagger compact category of mixed states and operations [27];

- Environment structures, an axiomatic substitute for the CPM-construction which proved to be particularly useful in the derivation of quantum protocols [7, 12].

It is well known that assuming compactness imposes finite dimension when exporting these results to the Hilbert space model [16. This paper introduces variations of each the above two results that rely on dagger structure alone, and in the presence of compactness reduce to the above ones. Hence, these variations accommodate interpretation not just in the dagger compact category of finite dimensional Hilbert spaces and linear maps, but also in the dagger category of Hilbert spaces of arbitrary dimension and continuous linear maps. We show:

- that the generalized CPM-construction indeed corresponds to the usual definitions of infinite-dimensional quantum information theory;

- that the direct correspondence between the CPM-construction and environment structure (up to the so-called doubling axiom) still carries trough.

The next two sections each discuss one of our two variations in turn. 
Earlier work The variation of the CPM-construction relying solely on dagger structure was already publicized by one of the authors as a research report [5]. Here we relate that construction to the usual setting of infinite-dimensional quantum information theory, thereby justifying it in terms of the usual model. An earlier version of this construction appeared in conference proceedings [8]. Whereas composition is not always well-defined there, it did have the advantage that the output category of the construction was automatically small if the input was. The construction here is closer to [5], and has the advantage that it is rigorously well-defined, but the disadvantage that the output category might be large. See the discussion after Proposition 4 and Remark 8 . Additionally, we generalise the construction further than [8], to braided monoidal categories that are not necessarily symmetric.

Related work While there are previous results dealing with the transition to a noncompact setting in some way or another, e.g. 1, 16, 17, what is particularly appealing about the results in this paper is that they still allow the diagrammatic representations of braided monoidal categories [21, 28.

Future work Classical information can be modelled in categorical quantum mechanics using so-called classical structures [11, 10, 3. It is not clear whether these survive CPM-like constructions; see also [18. The environment structures of Section 3 could be a useful tool in this investigation.

\section{Complete positivity}

Compact categories and their graphical calculus originated in [23, 24]. For a gentle introduction to dagger (compact) categories [2] and their graphical calculus [27, we refer to 9]. We now recall the CPM-construction [27, that, given a dagger compact category $\mathbf{C}$, produces a new dagger compact category $\operatorname{CPM}(\mathbf{C})$ as follows. When wires of both types $A$ and $A^{*}$ arise in one diagram, we will decorate them with arrows in opposite directions. When possible we will suppress coherence isomorphisms in formulae. Finally, recall that (_)* reverses the order of tensor products, so $f_{*}$ has type $A^{*} \rightarrow B^{*} \otimes C^{*}$ when $f: A \rightarrow C \otimes B[27$.

- The objects of $\operatorname{CPM}(\mathbf{C})$ are the same as those of $\mathbf{C}$.

- The morphisms $A \rightarrow B$ of $\mathrm{CPM}(\mathbf{C})$ are those morphisms of $\mathbf{C}$ that can be written in the form $\left(1 \otimes \eta^{\dagger} \otimes 1\right)\left(f_{*} \otimes f\right): A^{*} \otimes A \rightarrow B^{*} \otimes B$ for some morphism $f: A \rightarrow X \otimes B$ and object $X$ in $\mathbf{C}$.

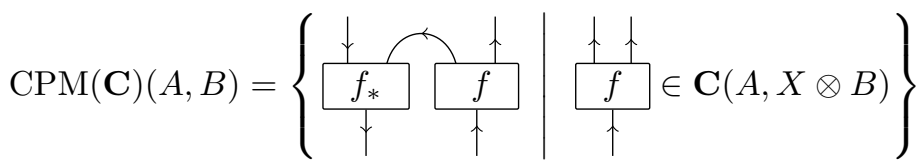


We call $X$ the ancillary system of $\left(1 \otimes \eta^{\dagger} \otimes 1\right)\left(f_{*} \otimes f\right)$, and $f$ its Kraus morphism; these representatives are not unique.

- Identities are inherited from $\mathbf{C}$, and composition is defined as follows.

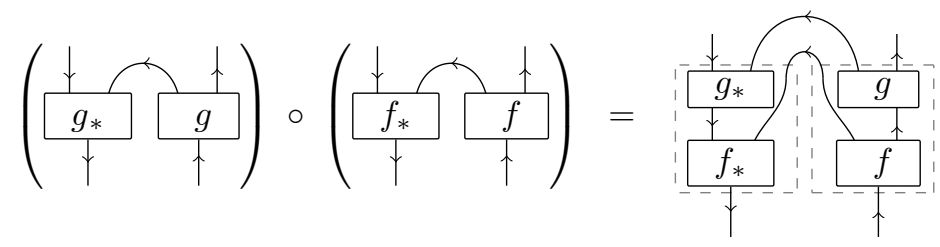

- The tensor unit $I$ and the tensor product of objects are inherited from $\mathbf{C}$, and the tensor product of morphisms is defined as follows.

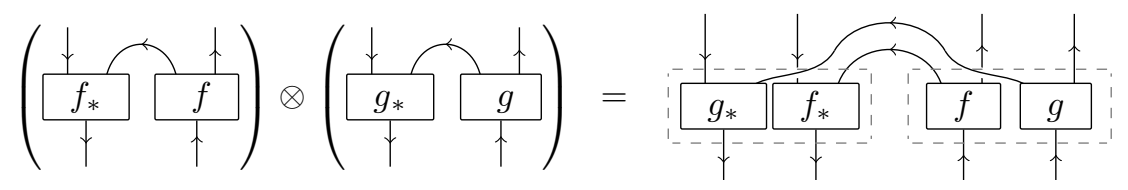

- The dagger is defined as follows.

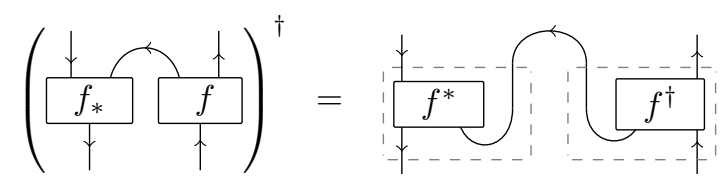

- Finally, the cup $\eta_{A}: I \rightarrow A^{*} \otimes A$ in $\operatorname{CPM}(\mathbf{C})$ is given by $\left(\eta_{A}\right)_{*} \otimes \eta_{A}=$ $\eta_{A} \otimes \eta_{A}$ in $\mathbf{C}$ (i.e. with ancillary system $I$ and Kraus morphism $\eta_{A}$ in $\mathbf{C}$ ).

If $\mathbf{C}$ is the dagger compact category FHilb of finite-dimensional Hilbert spaces and linear maps, then $\mathrm{CPM}(\mathbf{F H i l b})$ is precisely the category of finite-dimensional Hilbert spaces and completely positive maps [27. To come closer to the traditional setting, we may identify the objects $H$ of $\mathrm{CPM}(\mathbf{F H i l b})$ with their algebras of operators $B(H)$.

The notion of complete positivity makes perfect sense for normal linear maps between von Neumann algebras $B(H)$ for Hilbert space $H$ of arbitrary dimension. We now present a $\mathrm{CP}^{\infty}$-construction that works on dagger monoidal categories that are not necessarily compact, and that reduces to the previous construction in the compact case. Subsequently we prove that applying this construction to the category of Hilbert spaces indeed results in the traditional completely positive maps as morphisms.

The idea behind the following construction is to rewrite the morphisms

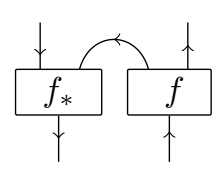

as

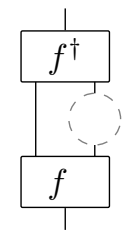


in a form not using compactness. Composition becomes plugging the hole. For this to be well-defined, we need to take the Kraus morphism $f$ as a representative seriously.

Definition 1. Define an equivalence relation on $\bigcup_{X \in \mathbf{C}} \mathbf{C}(A, X \otimes B)$ by setting $f \sim g$ when

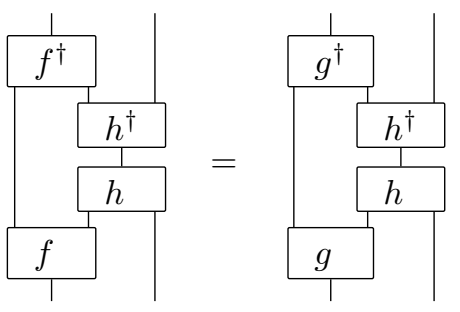

for all $h: B \otimes Y \rightarrow Z$ in a dagger monoidal category $\mathbf{C}$.

Lemma 2. If $f: A \rightarrow X \otimes B$ and $v: X \rightarrow Y$ in a dagger monoidal category satisfy $v^{\dagger} v=1$, then $f \sim(v \otimes 1) f$.

Proof. Immediate from the definition of the equivalence relation.

Definition 3. For a dagger monoidal category $\mathbf{C}$, define a new category $\mathrm{CP}^{\infty}(\mathbf{C})$ as follows.

- The objects of $\mathrm{CP}^{\infty}(\mathbf{C})$ are those of $\mathbf{C}$.

- The morphisms $A \rightarrow B$ of $\mathrm{CP}^{\infty}(\mathbf{C})$ are equivalence classes of morphisms $f: A \rightarrow C \otimes B$ of $\mathbf{C}$.

$$
\mathrm{CP}^{\infty}(\mathbf{C})(A, B)=\{f \in \mathbf{C}(A, X \otimes B) \mid X \in \mathbf{C}\} / \sim
$$

We call a representative $f$ a Kraus morphism and $X$ its ancillary system.

- The coherence isomorphism $A \rightarrow I \otimes A$ of $\mathbf{C}$ represents the identity on $A$ in $\mathrm{CP}^{\infty}(\mathbf{C})$, and composition is defined as follows.

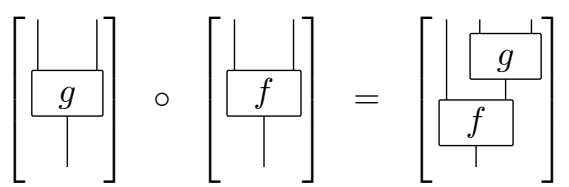

Proposition 4. If $\mathbf{C}$ is a dagger monoidal category, $\mathrm{CP}^{\infty}(\mathbf{C})$ is indeed a welldefined category.

Proof. Composition is well-defined, because if $f \sim f^{\prime}$ and $g \sim g^{\prime}$, then $\alpha^{\dagger}(1 \otimes$ g) $f \sim \alpha^{\dagger}\left(1 \otimes g^{\prime}\right) f^{\prime}$, where we write $\alpha$ for the coherence isomorphism $(X \otimes Y) \otimes$ $C \rightarrow X \otimes(Y \otimes C)$. Applying Lemma 2 to the same coherence isomorphism $\alpha$ shows that composition is associative. Applying Lemma 2 to the coherence isomorphisms $X \otimes I \rightarrow X$ and $I \otimes X \rightarrow X$ shows that pre- and post-composition with identities leaves morphisms invariant. 
Before we go on to show that $\mathrm{CP}^{\infty}(\mathbf{C})$ is a monoidal category, let us discuss size issues. In general, $\operatorname{CPM}(\mathbf{C})$ is just as large as $\mathbf{C}$, but this is less clear for $\mathrm{CP}^{\infty}(\mathbf{C})$. If $\mathbf{C}$ is small, then so is $\mathrm{CP}^{\infty}(\mathbf{C})$, because set-indexed unions of sets are again sets [15. If $\mathbf{C}$ is essentially small, meaning that it is dagger equivalent to a small category, then so is $\mathrm{CP}^{\infty}(\mathbf{C})$, by Lemma 2 . If $\mathbf{C}$ is locally small, it is not clear that $\mathrm{CP}^{\infty}(\mathbf{C})$ is again locally small. This does hold for large but locally small categories $\mathbf{C}$ such as Hilb and Rel, that are well-powered and have good notions of image [19] and rank: if a morphism $f: A \rightarrow X \otimes B$ preserves rank, its image can at most be as large as $A$, so we may restrict to ancillary systems $X$ that are subobjects of $A$, which constitute a set. Compare this to the existence of a minimal Stinespring dilation, or the fact that it is enough to check $n$-positivity to verify that a map between $n$-dimensional systems is completely positive by Choi's theorem [25].

Proposition 5. If $\mathbf{C}$ is a dagger braided monoidal category, then $\mathrm{CP}^{\infty}(\mathbf{C})$ is a braided monoidal category. The tensor unit I and tensor products of objects are as in $\mathbf{C}$, and the tensor product of morphisms is defined as follows.

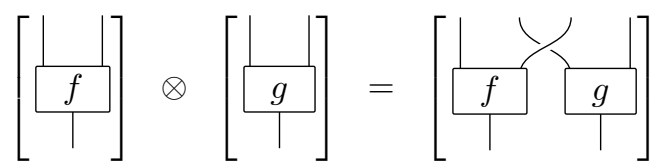

Moreover, if $\mathbf{C}$ is dagger symmetric monoidal, then $\mathrm{CP}^{\infty}(\mathbf{C})$ is symmetric monoidal.

Proof. Write $\alpha_{A, B, C}:(A \otimes B) \otimes C \rightarrow A \otimes(B \otimes C), \lambda_{A}: I \otimes A \rightarrow A$, and $\rho_{A}: A \otimes I \rightarrow A$ for the coherence isomorphisms of $\mathbf{C}$, and $\sigma_{A, B}: A \otimes B \rightarrow B \otimes A$ for the braiding. The coherence isomorphisms in $\mathrm{CP}^{\infty}(\mathbf{C})$ are

$\alpha_{A, B, C}=\left[\lambda_{A \otimes(B \otimes C)} \alpha_{A, B, C}\right], \quad \lambda_{A}=\left[1_{I \otimes A}\right], \quad \rho_{A}=\left[\sigma_{A, I}\right], \quad \sigma_{A, B}=\left[\lambda_{B \otimes A} \sigma_{A, B}\right]$.

Verifying the triangle, pentagon, and hexagon laws is laborious but straightforward, and repeatedly uses Lemma 2 and $\lambda_{I}=\rho_{I}$ [22, 1.1]. Similarly, $\mathrm{CP}^{\infty}(\mathbf{C})$ inherits symmetry from $\mathbf{C}$ :

$$
\begin{aligned}
\sigma_{A, B} \sigma_{B, A} & =\left[\lambda_{B \otimes A} \sigma_{A, B}\right] \circ\left[\lambda_{A \otimes B} \sigma_{B, A}\right] \\
& =\left[\left(1_{I \otimes I} \otimes \sigma_{B, A}\right) \alpha_{I, I, B \otimes A}^{\dagger} \lambda_{I \otimes(B \otimes A)}\left(1_{I} \otimes \sigma_{A, B}\right) \lambda_{A \otimes B}\right] \\
& =\left[\alpha_{I, I, A \otimes B}^{\dagger} \lambda_{I \otimes(A \otimes B)} \lambda_{A \otimes B}\right] \\
& =\left[\lambda_{A \otimes B}\right] \\
& =1_{A \otimes B},
\end{aligned}
$$

where the third equation follows from $\sigma_{A, B} \sigma_{B, A}=1_{A \otimes B}$ in $\mathbf{C}$, and the fourth equation follows from an application of Lemma 2 to $\rho_{I}: I \otimes I \rightarrow I$.

Notice that $\mathrm{CP}^{\infty}(\mathbf{C})$ does not obviously have a dagger, even though $\mathbf{C}$ does. Together with the fact that $\mathrm{CP}^{\infty}(\mathbf{C})$ is not obviously locally small when $\mathbf{C}$ is, this suggests that the $\mathrm{CP}^{\infty}$-construction is not straightforwardly a monad on dagger braided monoidal categories. 
Proposition 6. If $\mathbf{C}$ is a dagger compact category, then $\mathrm{CPM}(\mathbf{C})$ and $\mathrm{CP}^{\infty}(\mathbf{C})$ are isomorphic dagger symmetric monoidal categories. Hence if $\mathbf{C}$ is a dagger compact category, then so is $\mathrm{CP}^{\infty}(\mathbf{C})$.

Proof. The isomorphism $\mathrm{CPM}(\mathbf{C}) \rightarrow \mathrm{CP}^{\infty}(\mathbf{C})$ is given by $A \mapsto A$ on objects, and acts as follows on morphisms.

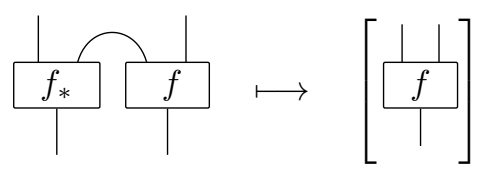

This assignment is clearly invertible. To see that it gives a well-defined functor preserving daggers and symmetric monoidal structure takes light work. See [5, Lemma 2.2] for details.

If canonical Kraus morphisms are available, as in Hilb and Rel, we may use an alternative description of $\mathrm{CP}^{\infty}(\mathbf{C})$ that does not use equivalence relations, as in [8]. The following corollary spells this out for compact $\mathbf{C}$, such as Rel.

Corollary 7. In a dagger compact category, $f \sim g$ if and only if

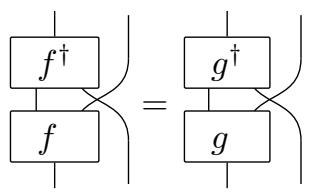

for $f: A \rightarrow X \otimes B$ and $g: A \rightarrow Y \otimes B$.

Proof. By definition, $f \sim g$ in $\mathbf{C}$ means precisely that $[f]=[g]$ in $\mathrm{CP}^{\infty}(\mathbf{C})$. Via the isomorphism of Proposition 6, elementary graphical manipulation then yields the statement.

Remark 8. One might try, as in 8 , to define $\mathrm{CP}^{\infty}(\mathbf{C})$ as a monoidal subcategory of $\mathbf{C}$ as follows.
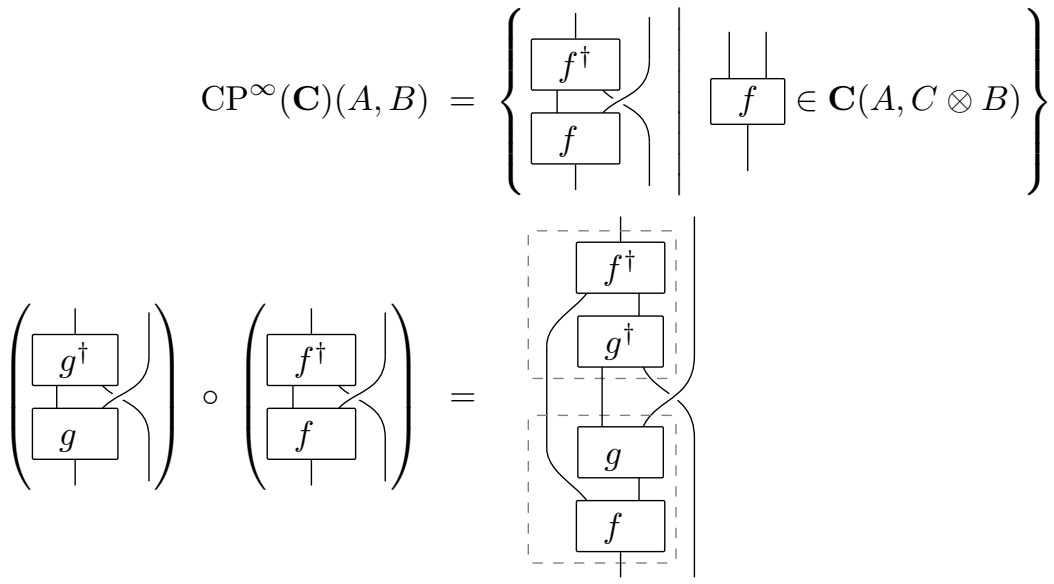
That is, the homset $\mathrm{CP}^{\infty}(\mathbf{C})(A, B)$ is a subset of $\mathbf{C}(A \otimes B, A \otimes B)$, and the equivalence relation $\sim$ is simply absorbed by the set comprehension notation. However, it is unclear that this composition is well-defined. Clearly the composition of morphisms $F: A \rightarrow B$ and $G: B \rightarrow C$ does not depend on the chosen representative $g: B \rightarrow Y \otimes C$ in $G=\left(g^{\dagger} \otimes 1\right)(1 \otimes \sigma)(g \otimes 1)$. But the composition does in general depend on the choice of representative of $F$.

The category Hilb has two properties that make this composition welldefined nevertheless. First, it is monoidally well-pointed: if $f(a \times b)=g(a \otimes b)$ for all $a: I \rightarrow A$ and $b: I \rightarrow B$, then $f=g: A \otimes B \rightarrow C$ [3]. Second, it is enriched over Banach spaces: by choosing orthonormal bases, any morphism $f: A \rightarrow B$ can be written in 'matrix form' as $f=\sum_{i} b_{i} a_{i}^{\dagger}$ for $a_{i}: I \rightarrow A$ and $b_{i}: I \rightarrow B$, and this (possibly infinite) sum respects composition and tensor products. Using these properties one sees that $\left(1 \otimes b^{\dagger}\right) G F(1 \otimes a)$ is independent of the chosen representative of $F$, and hence so is $G F$.

We now embark on showing that the $\mathrm{CP}^{\infty}$-construction is not just formal manipulation of diagrams that just happens to coincide with the traditional setting in the case of finite-dimensional Hilbert spaces.

To do so, we first recall the definition of quantum operations in the Heisenberg picture, as it is usually stated in infinite-dimensional quantum information theory 20. A function $\varphi: A \rightarrow B$ between von Neumann algebras is unital when $\varphi(1)=1$, and positive when for each $a \in A$ there is $b \in B$ such that $\varphi\left(a^{*} a\right)=b^{*} b$. When it is linear, the function $\varphi$ is called normal when it preserves suprema of increasing nets of projections, or equivalently, when it is ultraweakly continuous. Finally, $\varphi$ is completely positive when $\varphi \otimes 1: A \otimes M_{n} \rightarrow B \otimes M_{n}$ is positive for all $n \in \mathbb{N}$, where $M_{n}$ is the von Neumann algebra of $n$-by- $n$ complex matrices. (See e.g. [29, 3.3] or [25, p26].)

Definition 9. A quantum operation is a normal completely positive linear map between von Neumann algebras 四 Hilbert spaces $H$ and quantum operations $B(H) \rightarrow B(K)$ form a category that we denote by QOperations.

To see that $\mathrm{CP}^{\infty}(\mathbf{H i l b})$ is isomorphic to QOperations, we will rely on two classical theorems: Stinespring's dilation theorem, showing that quantum operations can be written as *-homomorphisms on larger algebras, and Dixmier's structure theorem for normal *-homomorphisms. A *-homomorphism is a linear map $\pi: A \rightarrow B$ between $\mathrm{C}^{*}$-algebras that satisfies $\pi(a b)=\pi(a) \pi(b)$ and $\pi\left(a^{*}\right)=\pi(a)^{*}$.

Let us emphasize that the following results hold for arbitrary Hilbert spaces, not just separable ones.

Theorem 10 (Stinespring). Let $A$ be a von Neumann algebra. For any normal completely positive linear map $\varphi: A \rightarrow B(H)$, there exist a unital normal *-

\footnotetext{
${ }^{1}$ Quantum operations $\varphi$ are usually also taken to be subunital, that is, satisfying $\varphi\left(1_{H}\right) \leq$ $1_{K}$. We do not require this for the same reason as the original CPM-construction did not [26] Remark 6.1]. See also the discussion after Theorem 13

${ }^{2}$ Not every completely positive linear map between von Neumann algebras is normal. In fact, a positive linear map is normal if and only if it is weak-* continuous [13, 46.5].
} 
homomorphism $\pi: A \rightarrow B(K)$ and a continuous linear $v: H \rightarrow K$ such that $\varphi(a)=v^{\dagger} \pi(a) v$.

Proof. See [4, Theorem III.2.2.4].

Theorem 11 (Dixmier). Every normal *-homomorphism $\varphi: B(H) \rightarrow B(K)$ factors as $\varphi=\varphi_{3} \varphi_{2} \varphi_{1}$ for:

- an ampliation $\varphi_{1}: B(H) \rightarrow B\left(H \otimes H^{\prime}\right): f \mapsto f \otimes 1_{H^{\prime}}$ for some Hilbert space $H^{\prime}$;

- an induction $\varphi_{2}: B\left(H \otimes H^{\prime}\right) \rightarrow B\left(K^{\prime}\right): f \mapsto p f p$ for the projection $p \in$ $B\left(H \otimes H^{\prime}\right)$ onto some Hilbert subspace $K^{\prime} \subseteq H \otimes H^{\prime}$;

- a spatial isomorphism $\varphi_{3}: B\left(K^{\prime}\right) \rightarrow B(K): f \mapsto u^{\dagger} f u$ for a unitary $u: K \rightarrow K^{\prime}$.

Proof. See [14, I.4.3] or [29, IV.5.5].

Corollary 12. A linear map $\varphi: B(H) \rightarrow B(K)$ is a quantum operation if and only if it is of the form $\varphi(f)=g^{\dagger}(f \otimes 1) g$ for some Hilbert space $H^{\prime}$ and continuous linear map $g: K \rightarrow H \otimes H^{\prime}$.

Proof. Given a quantum operation $\varphi$, combine Theorems 10 and 11 to get $\varphi(f)=v^{\dagger} u^{\dagger} p(f \otimes 1) p u v$; taking $g=p u v$ brings $\varphi$ into the required form.

Conversely, one easily checks directly via Definition 9 that a map $\varphi$ of the given form is indeed normal, and completely positive.

Theorem 13. $\mathrm{CP}^{\infty}($ Hilb) and QOperations are contravariantly isomorphic symmetric monoidal categories.

Proof. The objects are already equal. The isomorphism sends a morphism $H \rightarrow K$ of $\mathrm{CP}^{\infty}$ (Hilb) with Kraus morphism $g: H \rightarrow X \otimes K$ to the quantum operation $g^{\dagger}\left(1 \otimes_{\_}\right) g: B(K) \rightarrow B(H)$.

This prescription is clearly (contravariantly) functorial and preserves tensor products. We show that it is well-defined. Suppose that $g \sim h$. Then by Definition $1 . g^{\dagger}\left(1 \otimes f^{\dagger} f\right) g=h^{\dagger}\left(1 \otimes f^{\dagger} f\right) h$; that is, the functions $g^{\dagger}\left(1 \otimes \_\right) g$ and $h^{\dagger}\left(1 \otimes \_\right) h$ are equal on positive elements $f^{\dagger} f$ of $B(K)$. But then these linear maps coincide on all of $B(K)$ because that $\mathrm{C}^{*}$-algebra is spanned by its positive elements.

Finally, this monoidal functor is invertible because of Theorem 10 .

We end this section by discussing quantum channels, that are usually defined as unital quantum operations; preserving units is the equivalent in the Schrödinger picture of preserving traces in the Heisenberg picture. If $\varphi(1)=1$ 
in Stinespring's Theorem 10, then $v^{\dagger} v=1$, i.e. $v$ is an isometry. This holds in the abstract, too. A morphism in $\operatorname{CPM}(\mathbf{C})$ has this property when

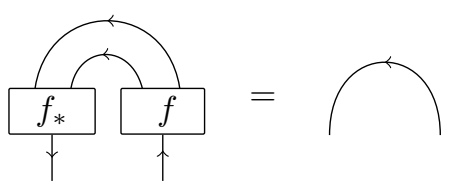

Via the analogy of equation (1), this means for morphisms in $\mathrm{CP}^{\infty}(\mathbf{C})$ that any Kraus morphism is an isometry. This is indeed independent of the Kraus morphism, for if $f \sim g$ for $f: A \rightarrow X \otimes B$ and $g: A \rightarrow Y \otimes B$, then $f^{\dagger} f=g^{\dagger} g$ by applying Definition 1 to $h=\rho_{B}: B \otimes I \rightarrow B$.

\section{Environment structures}

This section investigates when a given category is of the form $\mathrm{CP}^{\infty}(\mathbf{C})$ by generalizing [7, 12].

Definition 14. An environment structure for a dagger monoidal category $\mathbf{C}$ is a monoidal category $\widehat{\mathbf{C}}$ with the same objects, together with a strict monoidal functor $F: \mathbf{C} \rightarrow \widehat{\mathbf{C}}$ with $F(A)=A$, and for each object $A$ a morphism $\top_{A}: A \rightarrow$

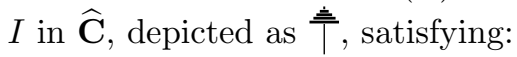

(a) We have $T_{I}=1_{I}$, and for all objects $A$ and $B$ : $\widehat{\mathbf{C}} ;$

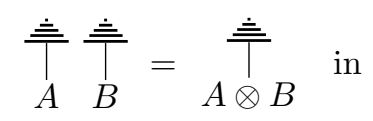

(b) Maps $f: A \rightarrow X \otimes B$ and $g: A \rightarrow Y \otimes B$ in $\mathbf{C}$ are equivalent if and only

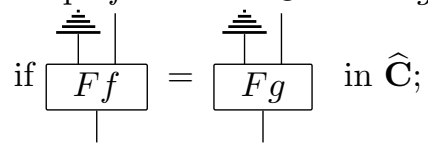

(c) For each $\widehat{f} \in \widehat{\mathbf{C}}(A, B)$ there is $f \in \mathbf{C}(A, X \otimes B)$ such that

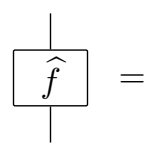

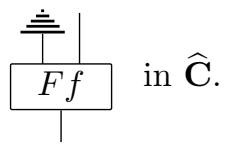

If the functor $F$ is faithful, we call the environment structure faithful.

As Theorem 16 below makes precise, the category Hilb has an environment structure, but the functor $F:$ Hilb $\rightarrow \mathrm{CP}^{\infty}$ (Hilb) identifies global phases and is therefore not faithful. 
Intuitively, if we think of the category $\mathbf{C}$ as consisting of pure states, then the supercategory $\widehat{\mathbf{C}}$ consists of mixed states. The maps $T$ 'ground' a system within the environment. Condition (c) then reads that every mixed state can be seen as a pure state in an extended system; the lack of knowledge carried in the ancillary system represents the variables relative to which we mix.

Having an environment structure is a sufficient condition for $\widehat{\mathbf{C}}$ to be of the form $\mathrm{CP}^{\infty}(\mathbf{C})$.

Theorem 15. If a dagger braided monoidal category $\mathbf{C}$ comes with an environment structure, then there exists an isomorphism $\mathrm{CP}^{\infty}(\mathbf{C}) \rightarrow \widehat{\mathbf{C}}$ of monoidal categories.

Proof. Define $\xi: \mathrm{CP}^{\infty}(\mathbf{C}) \rightarrow \widehat{\mathbf{C}}$ by setting $\xi(A)=A$ on objects, and

$$
\xi\left(\left[\begin{array}{c}
\perp \\
\hline f \\
\hline \mid
\end{array}\right]\right)=\underset{\underline{\underline{\underline{\underline{\prime}}}}}{F f}
$$

on morphisms. This map is well-defined and injective by Definition 14(b), and is surjective by Definition [14(c). It preserves composition and monoidal structure

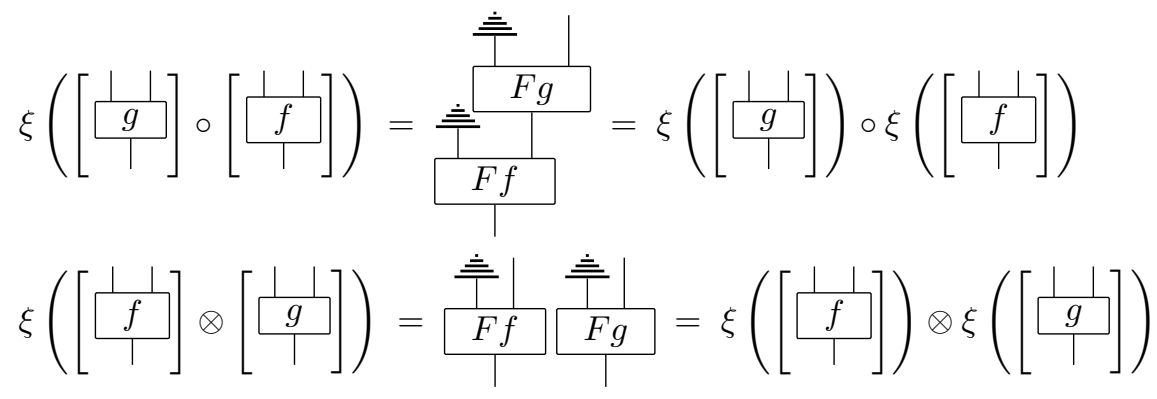

because $F$ is a strict monoidal functor and by Definition 14(a). It also clearly preserves identities. Thus $\xi$ is an isomorphism of monoidal categories.

Next we consider necessary conditions for a category to be of the form $\mathrm{CP}^{\infty}(\mathbf{C})$. If $\mathbf{C}$ is a dagger braided monoidal category, there is a canonical strict monoidal functor $F: \mathbf{C} \rightarrow \mathrm{CP}^{\infty}(\mathbf{C})$, given by $A \mapsto A$ on objects and by $f \mapsto\left[\lambda^{\dagger} f\right]$ on morphisms.

Theorem 16. Let $\mathbf{C}$ be a dagger braided monoidal category. Then $\widehat{\mathbf{C}}=\mathrm{CP}^{\infty}(\mathbf{C})$ with the canonical functor $F: \mathbf{C} \rightarrow \widehat{\mathbf{C}}$ and $\top_{A}=\left[\rho_{A}^{\dagger}\right] \in \widehat{\mathbf{C}}(A, I)$ is an environment structure.

Proof. Definition 14(a) and (c) are automatically satisfied, using Lemma 2 and the fact that $\lambda_{I}=\rho_{I}$. Let $f \in \mathbf{C}(A, X \otimes B)$ and $g \in \mathbf{C}(A, Y \otimes B)$. Unfolding definitions, we see that

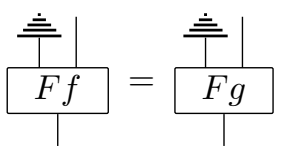


in $\widehat{\mathbf{C}}$ comes down to $f \sim g$ in C. Hence Definition [14(b) is satisfied.

Finally, we study when environment structures are faithful. For faithful environment structures, we may replace the functor $F$ by its image $\mathbf{D}$, which is a monoidal subcategory of $\mathrm{CP}^{\infty}(\mathbf{C})$. This is how Definition 14 relates to its namesake that was previously defined for the case when $\mathbf{C}$ is compact 12$]^{3}$ But if $\mathbf{C}$ is not compact, it is not clear whether $\mathbf{D}$ has a dagger, and thus whether Theorem 16 would still hold. To make the comparison, for the rest of this article we will assume that $\mathbf{C}$ is compact. We may think of $\mathbf{D}$ as a double of $\mathbf{C}$. The next definition makes this precise.

Definition 17. A dagger monoidal category satisfies the doubling axiom when

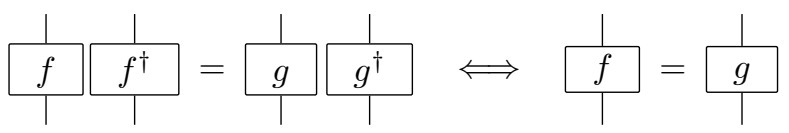

for all parallel morphisms $f$ and $g$.

Proposition 18. If $\mathbf{C}$ is a dagger compact category, then the canonical functor $F: \mathbf{C} \rightarrow \mathrm{CP}^{\infty}(\mathbf{C})$ is faithful if and only if $\mathbf{C}$ satisfies the doubling axiom.

Proof. Let $f, g \in \mathbf{C}(A, B)$, and suppose that $F(f)=F(g)$, that is, $\lambda^{\dagger} f \sim \lambda^{\dagger} g$. By Corollary 7 this is equivalent to,

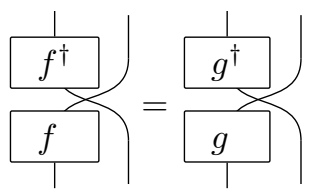

Naturality of the braiding shows this to be equivalent to $f \otimes f^{\dagger}=g \otimes g^{\dagger}$. This implies $f=g$ precisely when $\mathbf{C}$ satisfies the doubling axiom.

Recall that a dagger compact category $\mathbf{C}$ satisfies the so-called preparationstate agreement axiom [6] when $f f^{\dagger}=g g^{\dagger}$ implies $f=g$ for all morphisms $f$ and $g$ with domain $I$ :

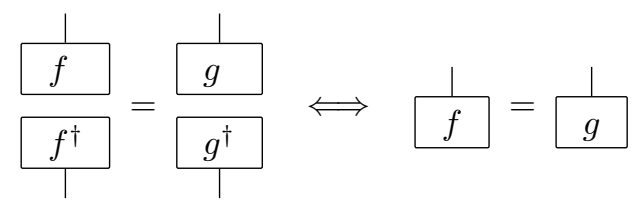

\footnotetext{
${ }^{3}$ The environment structures of [8] are precisely the faithful ones of Definition 14] Definition 17 below also differs from [8] to match this.
} 
So if $\mathbf{C}$ is a dagger compact category, then $\operatorname{CPM}(\mathbf{C})$ satisfies the preparationstate agreement axiom when

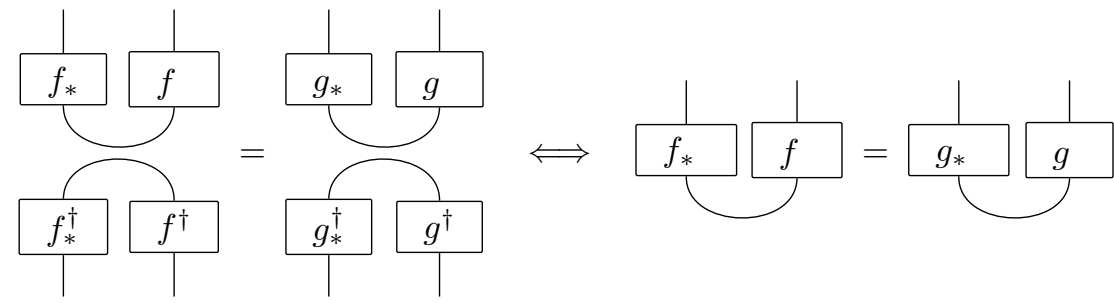

for all morphisms $f$ and $g$ with a common domain. The preparation-state agreement axiom (for dagger compact $\mathbf{C}$ ) is equivalent to the doubling axiom (for C) [5, Section 3]. However, the doubling axiom has an advantage over the preparation-state agreement axiom in that Proposition 18 can work when $\mathbf{C}$ is not compact, too, with the condition of Corollary 17. Moreover, the doubling axiom is stronger than the preparation-state axiom in the sense of the following proposition.

Proposition 19. If a dagger compact category $\mathbf{C}$ satisfies the doubling axiom, then $\operatorname{CPM}(\mathbf{C})$ satisfies the preparation-state agreement axiom.

Proof. Let $f$ and $g$ be morphisms with a common domain. Elementary graphical manipulations yield:

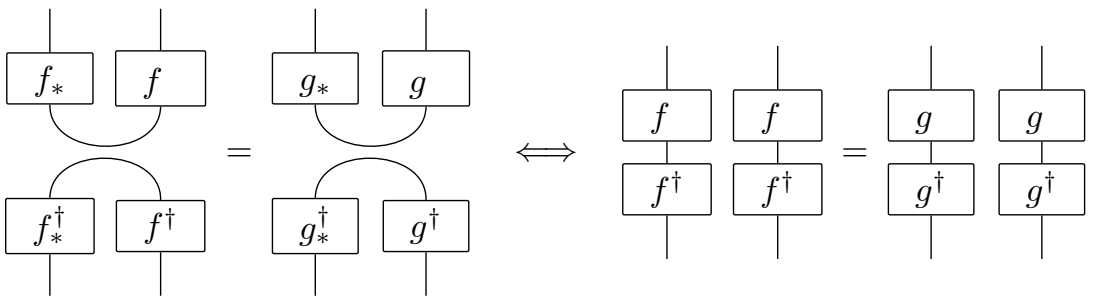

By the doubling axiom for $\mathbf{C}$, the latter is equivalent to $f f^{\dagger}=g g^{\dagger}$. Finally, by using graphical manipulation again, we obtain:

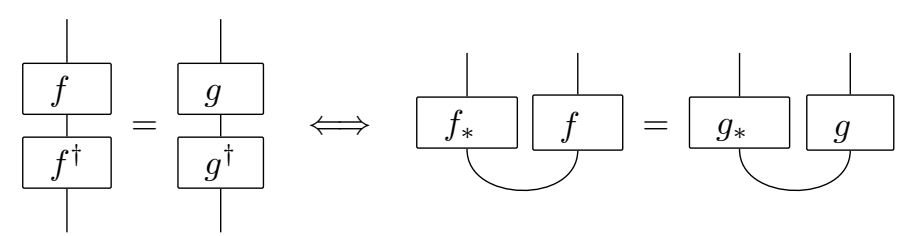

Hence $\operatorname{CPM}(\mathbf{C})$ satisfies the state-preparation agreement axiom.

\section{References}

[1] Samson Abramsky, Richard Blute, and Prakhash Panangaden. Nuclear and trace ideals in tensored *-categories. Journal of Pure and Applied Algebra, 143:3-47, 1999. 
[2] Samson Abramsky and Bob Coecke. A categorical semantics of quantum protocols. In Logic in Computer Science 19, pages 415-425. IEEE Computer Society, 2004.

[3] Samson Abramsky and Chris Heunen. $\mathrm{H}^{*}$-algebras and nonunital Frobenius algebras: first steps in infinite-dimensional categorical quantum mechanics. Clifford Lectures, AMS Proceedings of Symposia in Applied Mathematics, $71: 1-24,2012$.

[4] Bruce Blackadar. Operator algebras: theory of $C^{*}$-algebras and von Neumann algebras. Springer, 2006.

[5] Bob Coecke. Complete positivity without positivity and without compactness. Technical Report PRG-RR-07-05, Oxford University Computing Laboratory, 2007.

[6] Bob Coecke. De-linearizing linearity: Projective quantum axiomatics from strong compact closure. Electronic Notes in Theoretical Computer Science, 170:49-72, 2007.

[7] Bob Coecke. Axiomatic description of mixed states from selinger's cpmconstruction. Electronic Notes in Theoretical Computer Science, 210:3-13, 2008 .

[8] Bob Coecke and Chris Heunen. Pictures of complete positivity in arbitrary dimension. In Quantum Physics and Logic VIII, volume 95 of Electronic Proceedings in Theoretical Computer Science, pages 27-35, 2011.

[9] Bob Coecke and Éric O. Paquette. Categories for the practicing physicist. In B. Coecke, editor, New Structures for Physics, Lecture Notes in Physics, pages 167-271. Springer, 2011.

[10] Bob Coecke, Éric O. Paquette, and Duško Pavlović. Classical and quantum structuralism. In S. Gay and I. Mackie, editors, Semantic Techniques in Quantum Computation, pages 29-69. Cambridge University Press, 2010.

[11] Bob Coecke and Duško Pavlović. Quantum measurements without sums. In Mathematics of Quantum Computing and Technology, pages 559-596. Taylor and Francis, 2007.

[12] Bob Coecke and Simon Perdrix. Environment and classical channels in categorical quantum mechanics. In CSL'10/EACSL'10, pages 230-244. Springer, 2010.

[13] John B. Conway. A course in operator theory. American Mathematical Society, 2000.

[14] Jacques Dixmier. Von Neumann algebras. North-Holland, 1969.

[15] Paul R. Halmos. Naive set theory. Van Nostrand, 1960. 
[16] Chris Heunen. Compactly accessible categories and quantum key distribution. Logical Methods in Computer Science, 4(4), 2008.

[17] Chris Heunen. An embedding theorem for Hilbert categories. Theory and Applications of Categories, 22(13):321-344, 2009.

[18] Chris Heunen and Sergio Boixo. Completely positive classical structures and sequentializable quantum protocols. In Quantum Physics and Logic VIII, volume 95 of Electronic Notes in Theoretical Computer Science, pages 91-101, 2011.

[19] Chris Heunen and Bart Jacobs. Quantum logic in dagger kernel categories. Order, 27(2):177-212, 2010.

[20] Alexander S. Holevo. Statistical structure of quantum theory. Springer, 2001.

[21] André Joyal and Ross Street. The geometry of tensor calculus I. Advances in Mathematics, 88:55-112, 1991.

[22] André Joyal and Ross Street. Braided tensor categories. Advances in Mathematics, 102:20-78, 1993.

[23] G. Max Kelly. Many-variable functorial calculus I. In G. M. Kelly, M. Laplaza, G. Lewis, and S. Mac Lane, editors, Coherence in Categories, volume 281 of Lecture Notes in Mathematics, pages 66-105. SpringerVerlag, 1972.

[24] G. Max Kelly and Miguel L. Laplaza. Coherence for compact closed categories. Journal of Pure and Applied Algebra, 19:193-213, 1980.

[25] Vern Paulsen. Completely bounded maps and operators algebras. Cambridge University Press, 2002.

[26] Peter Selinger. Towards a quantum programming language. Mathematical Structures in Computer Science, 14(4):527-587, 2004.

[27] Peter Selinger. Dagger compact closed categories and completely positive maps. In Quantum Programming Languages, volume 170 of Electronic Notes in Theoretical Computer Science, pages 139-163. Elsevier, 2007.

[28] Peter Selinger. A survey of graphical languages for monoidal categories. In New Structures for Physics, volume 813 of Lecture Notes in Physics, pages 289-355. Springer, 2010.

[29] Masamichi Takesaki. Theory of Operator Algebra I. Encyclopaedia of Mathematical Sciences. Springer, 1979. 